\title{
The vocabulary of manga: Visual morphology in dialects of Japanese Visual Language
}

\author{
Neil Cohn *, Sean Ehly \\ Center for Research in Language, University of California, San Diego, United States \\ Received 1 August 2015; received in revised form 19 November 2015; accepted 22 November 2015
}

\begin{abstract}
The visual representations of non-iconic elements in comics of the world often take diverse and interesting forms, such as how characters in Japanese manga get bloody noses when lustful or have bubbles grow out their noses when they sleep. We argue that these graphic schemas belong to a larger "visual vocabulary" of a "Japanese Visual Language" used in the visual narratives from Japan. Our study first described and categorized 73 conventionalized graphic schemas in Japanese manga, and we then used our classification system to seek preliminary evidence for differences in visual morphology between the genres of shonen manga (boys' comics) and shojo manga (girls' comics) through a corpus analysis of 20 books. Our results find that most of these graphic schemas recur in both genres of manga, and thereby provide support for the idea that there is a larger Japanese Visual Language that pervades across genres. However, we found different proportions of usage for particular schemas within each genre, which implies that each genre constitutes their own "dialect" within this broader system.
\end{abstract}

(C) 2015 Elsevier B.V. All rights reserved.

Keywords: Visual language; Japanese Visual Language; Visual morphology; Manga; Comics; Japan

\section{Introduction}

Though the visual narratives of comics have become ubiquitous as cross-cultural products of popular culture, the representations used in comics of different cultures often vary widely. For example, the way that emotions are conveyed in manga-the comics of Japan-might often seem unusual to those unfamiliar with its conventions: Characters get bloody noses when lustful, bubbles grow out their noses when people sleep, and characters rapidly transform into hyper-cartoony representations in times of high emotion (see Fig. 1, and Appendix 1). Just what is going on in these depictions?

Recent work has argued that conventionalized drawing systems, especially those found in comics around the world, constitute "visual languages" that are structured and comprehended in analogous ways to spoken and signed languages (Cohn, 2013b). Elements such as bloody noses and nose bubbles can be thought of as "visual morphemes" in the visual language used in manga. A subset of these elements have been called "emanata" in English (Walker, 1980; Abel and Madden, 2008). Forceville et al. (2014) also subdivided these elements into classes of "pictorial runes" for non-literal graphic elements, such as bloody noses or sleep bubbles (Forceville, 2005; Kennedy, 1982; Forceville et al., 2014), "pictograms" for stylized depictions originating outside of the visual language of comics, such as hearts or dollar signs,

\footnotetext{
* Corresponding author at: Center for Research in Language, University of California, San Diego, 9500 Gilman Dr. Dept. 0526, La Jolla, CA 92093-0526, United States. Tel.: +1 858822 0736; fax: +1 8588225097.

E-mail address: neilcohn@visuallanguagelab.com (N. Cohn).
} 


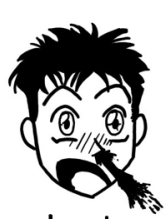

Lust

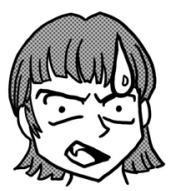

Shock or Exasperation
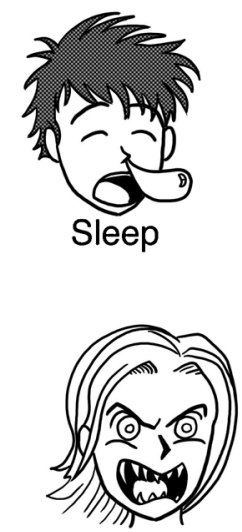

Anger

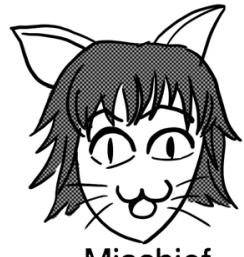

Mischief

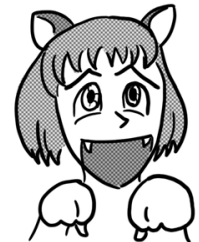

Begging

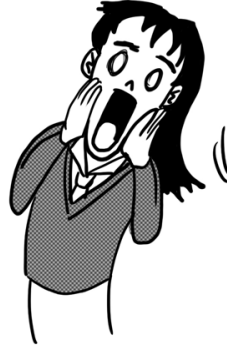

Shock

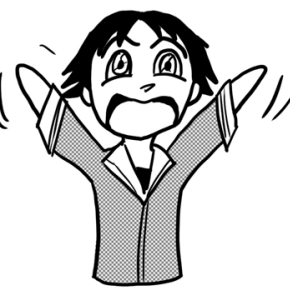

Loss of control

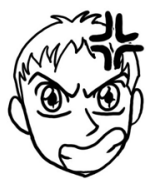

Anger or Irritation

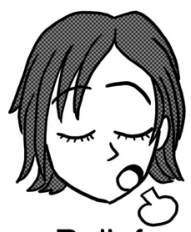

Relief

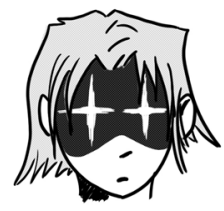

Glaring

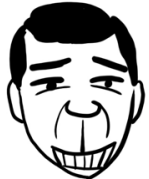

Dirty Thoughts

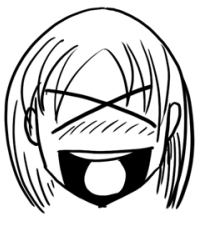

Excitement
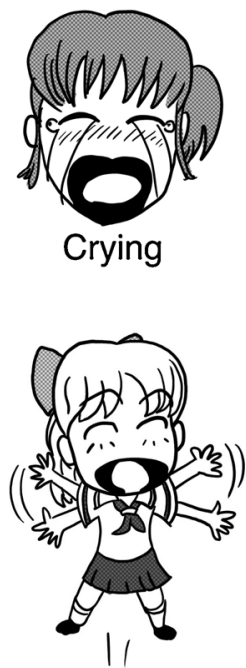

Shock

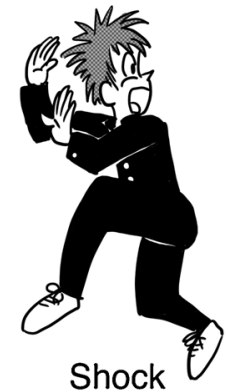

Super-deformation

(Chibi)

Fig. 1. Schematized representations of a selection of visual morphology from the Japanese Visual Language used in manga.

and "balloons" for carriers of text. Visual Language Theory (VLT) does not necessarily distinguish these particular subdivisions of visual morphemes because comics are merely one place in which visual languages appear, and the lines between the visual vocabulary used in comics and general "visual culture" are often blurred (both synchronically and diachronically) and frequently dependent on specific cultural contexts. Rather, VLT follows linguistics in categorizing visual morphemes in terms of their productive, combinatorial, and/or semiotic characteristics (discussed below).

Distinctions between these elements have also been made in Japanese-language manga scholarship. Visual morphemes are characterized within the broad class of kei yu ("metaphorical forms"), which breaks down into several subtypes (Natsume, 1997; Takekuma, 1995): Manpu ("manga specific signs") carry particular meanings, like bloody noses and sleep bubbles, while koka ("impact") convey elements like motion lines, zoom lines, and more general emotional and psychological states, including elements in an image's background (Takekuma, 1995). However, only some of these terms have gained traction in Japanese-language manga research. Again, VLT treats all such forms as visual morphemes.

This paper offers a preliminary investigation into the "visual vocabulary" used in "Japanese Visual Language" by assessing whether differences arise in the usage of these forms between the genres of shonen manga (boys' comics) and shojo manga (girls' comics). Most empirical studies examining the "visual vocabulary" of comics of the world have either examined a limited corpus of books (Forceville, 2005, 2011; Forceville et al., 2010; Feng and O'Halloran, 2012) or have looked at a limited range of "visual morphemes" (Abbott and Forceville, 2011; Shinohara and Matsunaka, 2009). 


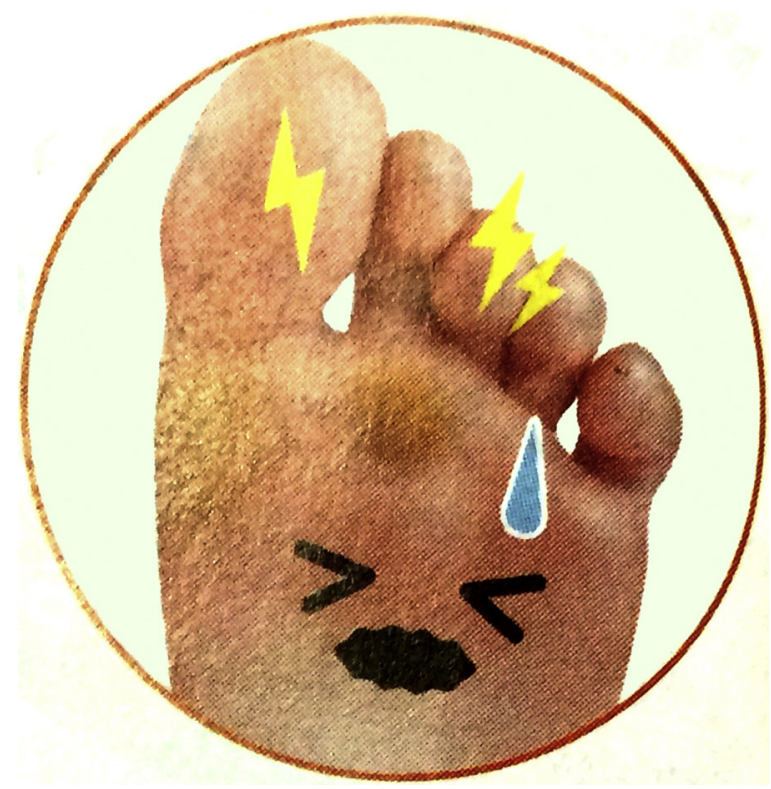

Fig. 2. Use of Japanese Visual Language morphology in an advertisement for foot pain.

However, in order to instigate an analysis of larger systems of representation than case studies (i.e., visual languages rather than idiolects) we need to address structures across multiple creators. Thus, we sought to go beyond these studies by coding 73 visual morphemes across ten books in each of these genres of manga, making it the largest corpus analysis to date examining these issues.

\section{Background}

Just what is meant by "visual language"? Humans use patterned ways of communicating in the visual-graphic form (i. e., drawing) just as they do in the verbal form (i.e., speaking). However, there is a terminological gap between these modalities with regards to the system employed in this process: we speak in a spoken language, but we draw in __? . The answer to filling this gap is a "visual language," and this broader theory (Cohn, 2013b) is integrated into contemporary theories of linguistics and cognition (e.g., Jackendoff, 2002). Support for this parallelism has come from both theory and from empirical findings of similar neural responses to manipulations of visual sequences as to sentences (e.g., Cohn et al., 2012, 2014).

In this paradigm, a visual vocabulary is understood in cognitive terms: graphic patterns are stored as schemas of formmeaning mappings in the long-term memory of their creators, similar to the way that verbal patterns are stored as schemas (words) in spoken languages of the world (Cohn, 2013b). To the extent that people might share the same cognitive patterns, we might say that they draw in a common visual language. Like other languages, visual languages are not universal, and thus they might differ across cultures-because patterns may be instantiated in the minds of groups of creators in different ways both between and within cultures.

Most manga are thus written in "Japanese Visual Language" (JVL), which contrasts from the visual languages used to create comics from other countries, like the United States or France. It is important to stress that this idea of "Japanese Visual Language" is not a synonym for "manga," just as "visual language" is not a synonym for "comics" more broadly. Japanese Visual Language describes the system of pictorial communication used by individuals that stereotypically appears in manga of the world (whether created by individuals within or outside of Japan), while "manga" is a sociocultural artefact that may or may not be actually written in JVL. That is, manga may be written in JVL alongside written Japanese, ${ }^{1}$ analogous to how books may be written solely in Japanese, though not all manga are written in JVL, nor are all books written in Japanese.

Indeed, JVL appears in many contexts that are not manga, especially in Japan. Take for example Fig. 2, excerpted from a catalogue of products found on a train in Japan. This advertisement was for a crème treating foot pain, which used

\footnotetext{
${ }^{1}$ Note that written languages are not "natural" languages and are also not "visual languages." Writing systems, including the various Japanese scripts, are a conversion of the natural verbal modality into the natural visual modality to create an unnatural (although culturally useful) mapping. Though writing systems thus share the same visual-graphic modality as natural visual languages, they are not classified as belonging to visual languages directly (see Cohn, 2013b for more details).
} 
Fig. 2 to depict this pain by adding no less than three visual morphemes from JVL to a photograph of a foot: lightning pain lines, a sweat drop for anxiety, and the $><$ for wincing eyes. This context is clearly outside of manga, yet uses the same visual vocabulary. Many of these same visual lexical items have also been co-opted into emoji for use in digital chatting. Insofar as "JVL" and "manga" are different things, the study of JVL thus does not merely apply to published works, whether within or outside of manga. Rather, JVL applies to the analysis of systematic graphic representations ranging from non-manga usage to children's drawings (Wilson, 1999, 2015) to professional manga creators and everything in between. However, we here analyze published works as the most prototypical and influential sample of JVL usage.

In the context of Visual Language Theory (Cohn, 2013b), the term "morpheme" is used to describe units of formmeaning pairs in the graphic system, analogous to the way that mappings of form (phonology) and meaning constitute the vocabularies of spoken languages (Jackendoff, 2002; Booij, 2010). We will use this term, along with "graphic schema," to describe units of meaningful visual representation (such as a heart shape), as well as more complex compositions of aggregate parts (such as whole faces, which themselves are built of eye, mouth, and nose morphemes) which may form perceptually salient meaningful units. This applies to conventionalized schemas that use various types of reference (Peirce, 1931)-iconic (faces), indexical (tails on word balloons), or symbolic (hearts). Ultimately, this nomenclature is not interested in drawing a hard equivalence between spoken and visual representations, or with specifically isolating the level of a "minimal" representation, as might have been the case in structuralist accounts of visual analysis (e.g., Gubern, 1972; Hünig, 1974; Koch, 1971; Nöth, 1990). Rather, we are here more concerned with systematic patterns of formmeaning relationships in the graphic domain, regardless of size or internal complexity, as stored in the memory of creators and readers of manga.

Our study had two aims. First, we sought to describe and categorize the visual morphemes used in the Japanese Visual Language found in manga, a selection of which is depicted in Fig. 1. Some work has attempted to classify the visual morphology used in comics and graphic novels using corpus analysis of stereotypical European and American comics (Forceville, 2011; Forceville et al., 2010). However, previous work has only examined a limited range of schemas used in manga (Abbott and Forceville, 2011; Cohn, 2010, 2013b; Shinohara and Matsunaka, 2009; Brenner, 2007; Takekuma, 1995). Thus, we here attempt to expand such observations to better describe the visual vocabulary used in manga specifically with regard to morphemes related to emotion.

Our second aim drew upon our previous claims that the generalized "Japanese Visual Language" used across authors of manga is instantiated in different "dialects" (Cohn, 2010, 2013b). Because visual language is a print culture not restricted by geographical boundaries to separate populations of "speakers," dialects here are demarcated by genres, since they separate different groups of people who interact with these systems. Thus, we used our classification system to seek preliminary evidence for differences in visual morphology between the genres of shonen manga (boys' comics) and shojo manga (girls' comics).

What do we mean by a "dialect" of visual language? As in spoken languages, the notion of a visual "dialect" is no different in cognitive status than a full "language"-they all reflect patterned visual-graphic expressions that are stored in the minds of their "speakers." If differences do appear between "Shonen JVL" and "Shojo JVL," these dialects would still exist on the same level as cognitive phenomena, though these systems may be perceived differently in their cultural status (i.e., one may be "marked" as stereotypical of the broader system more than another-a point worth investigating under a lens of "visual sociolinguistics").

We here use the term "dialect" to refer to systems that share an overarching similar structure (as part of a generalized, yet abstract, "Japanese Visual Language"), while still retaining patterns of variation that distinguish them as unique systems. This would be analogous to the conception that Kanto Japanese (the dialect(s) spoken in areas surrounding Tokyo) and Kansai Japanese (the dialect(s) spoken in areas surrounding Osaka) exist as dialects of the generalized "Japanese spoken language," which are similar across most dimensions of structure in ways that are mutually intelligible for speakers, though they differ across many structures (pronunciation, vocabulary, syntax, etc.). As in spoken languages, visual language dialects may also deviate from each other across all levels of structure, such as graphic structure (i.e., visual style), narrative structure, panel framing, page layout, and others (Cohn, 2013b). Certainly, any complete characterization of different "dialects" or "languages" would require analysis beyond just one dimension of structure, but we here specifically investigate differences in systems with regard to visual morphology as an initial foray into characterizing the similarities and differences between graphic systems. Thus, the questions here are: (1) to what degree might Shonen JVL and Shojo JVL share a common visual vocabulary (and thus belong to a common "Japanese Visual Language"), and (2) to what degree do we find variation in the patterns between these systems (thus constituting unique dialects)?

\section{The visual vocabulary of manga}

Our first task was to establish a visual vocabulary of the morphology used in Japanese Visual Language broadly. Like spoken languages, we can classify the vocabularies of visual languages as consisting of two general classes (Cohn, 2013b). "Open class" items in a lexicon are productive, and new elements can easily be created. In visual languages, 
these representations are often iconic (resembling their meaning), since an author can easily derive new patterns of ways to draw things based on what they look like in real life. For example, open class items often include the ways that authors draw characters' figures and body parts, since those elements are potentially variable and new patterns can easily be created when necessary (such as when a character has an attribute for which the drawer has no established pattern). "Closed class" lexical items are much harder to create, since they often rely on conventionalized meanings that are unavailable without the knowledge of what they mean, such as bloody noses to mean lust (an iconic representation with symbolic meaning). Our analysis included both types of morphology, though our primary focus is on "closed class" items.

Previous works that have examined the morphology in manga have either offered broad surveys of various visual morphemes (Cohn, 2010, 2013b; Takekuma, 1995; Natsume, 1997), have discussed the targeted usage of specific morphemes (Abbott and Forceville, 2011), have discussed deeper metaphoric qualities of a survey of morphemes (Shinohara and Matsunaka, 2009; Abbott and Forceville, 2011), or have tested the comprehension of various morphemes (Nakazawa, 2005; Kikuchi et al., 2005; Murata, 1994; Nakazawa, 1998). Though we aim here to provide a more extensive catalogue of JVL morphology, we make no claims of being exhaustive in this investigation either, and have limited our scope to visual morphemes that contain some sort of emotional salience. Additional, fairly ubiquitous visual morphemes have been excluded from this discussion, such as the diverse range of motion lines used in manga to show moving objects (Cohn, 2013b; McCloud, 1993, 1996) or the diversity of carriers of text, such as speech balloons or thought bubbles (Cohn, 2013a; Forceville et al., 2010). However, we did include "circumfixing" motion lines, which surround a figure to depict shaking, and "zoom lines," which draw focus to a character in a panel, because these morphemes relate to aspects of emotional salience. A full investigation of JVL morphology would need to include all such schemas, and thus we consider our study a preliminary effort towards this broader goal. Studies on motion lines and carriers of text are forthcoming.

We used the precedents in the literature in combination with an informal corpus study to establish a list of 73 visual morphemes and patterns used in JVL. A full listing of morphological forms along with examples appear in Appendix 1: Manga morphology, which is available online at http://www.visuallanguagelab.com/A/jvlmorphology.html.

In highlighting the similarities between verbal and visual systems, we previously argued that visual language morphology uses analogous "combinatorial strategies" as the morphology in verbal languages (Cohn, 2013b). For example, some morphemes may attach to another more dominant morpheme (affixation), morphemes may replace entirely or partially with another (suppletion), and some morphemes might repeat (reduplication). It is important to stress that this analogy between morphology in visual languages and verbal languages relates to functional similarities only. Affixes in visual language are not argued to be directly equivalent to affixes in verbal language in terms of the mechanisms that guide their comprehension. Rather, using affixation as an example, the claim is that both domains use a similar strategy of "attachment" in their combinatorial structure, whether or not those strategies are tied to a common cognitive process (although, see Cohn and Maher, 2015).

These distinctions about morphological structure can provide an overarching system to categorize the different types of visual morphemes in JVL. While most morphemes fit within these broad rubrics, some morphemes do not easily fall within such categories because of the differences in affordances between the verbal and graphic domains. It is therefore emphasized again that finding specific analogies between the verbal and visual domains is less important than describing how these form-meaning pairs operate in their own natural graphic modality.

\subsection{Affixation}

Affixation seems to be the most simple and prevalent way of combining visual morphemes, where one morpheme (the affix) attaches to another more dominant morpheme (a root) (Cohn, 2013b; Engelhardt, 2002). A clear affix might be the "carriers" that hold text, stereotypically speech balloons, thought bubbles, captions, and sound effects (Cohn, 2013a,b; Forceville et al., 2010). These morphemes must attach to a "root," such as a "speech balloon" attached to a "speaker." JVL has several interesting uses of carriers, though we here focus on two types particularly relevant for emotional representations: floating borderless carriers depicting laughter and "speech balloons" that appear as small cloudy puffs to depict heavy breathing.

Another class of affixes go "up" from a character's head (Cohn, 2013b; Forceville, 2011) and thus have been named "upfixes," i.e., they are visual morphemes that float above characters' heads. JVL uses several upfixes, including those found in American and European visual languages, like hearts, exclamation marks, and question marks. JVL sometimes places these upfixes in carriers that appear like speech balloons, though they do not represent speech. Other JVL upfixes include steam that looks like puffs of smoke for anger or frustration, or heat lines for anger, angled lines for exasperation (called "spikes" in Forceville, 2011), and others.

In addition to morphemes above the head, affixes in JVL attach to other parts of the head and face. For example, some morphemes appear on top of characters' foreheads or heads, such as a stylized vein to depict anger (Shinohara and Matsunaka, 2009), a single huge sweat-drop to depict anxiety, sweat drops surrounding the head (called "plewds" in 
Walker, 1980), or vertical shadows on the head to show gloom. See Fig. 1 for several examples. Noses are also prominent: Bloody noses depict lust, a giant bubble from the nose indicates sleep, and steam may emit from a nose like a burst of smoke. Mouths also have a range of morphemes, such as the more iconic drool, a "mushroom"-looking puff of smoke emitting from the mouth for a sign of relief, and fire spouting from the mouth to show anger. Some morphemes also appear next to eyes, such as a "spark" or "half-spark" appearing next to eyes to show attention, similar to the scopic deictic lines that may draw attention to characters' eyes (Cohn, 2013b). A white "glow" on a black background also may appear next to characters' eyes, their whole face, and even around their whole body. At present, it is unclear whether these types of affixes are each unique instances, or whether consistent placement across affixes creates additional classes of signs related to each body part (Foreheadfixes? Nosefixes?).

Finally, some affixes related to emotions use highly schematic representations of iconic phenomena. For example, in contrast to the giant singular sweat-drop on the forehead, characters may have several beads of sweat across a face to show anxiety. Vertical lines descending from eyes show streaks of tears, and small bubbles in the corners of eyes depict a character on the verge of crying. JVL also typically uses a highly stylized depiction of blush on cheeks for embarrassment, which can extend across an absent nose into a full flush of the face. Other shading can occur on the face that is not iconic though: Shadows or shading can occur on the whole face, over the eyes, around the eyes, or under the eyes to show a sense of doom, surprise, or anxiety. This shading has nothing to do with actual light sources, but rather with a character's emotional state.

\subsection{Suppletion}

Morphemes may also create meaning by replacing another morpheme, be it full suppletion (ex. go becomes went in past tense, replacing the whole word), partial suppletion (ex. teach becomes taught in past tense, retaining the onset "t"), or internal replacement through umlaut (ex. sing becomes sang in past tense, changing only the internal vowel). For example, Abbott and Forceville (2011) describe a peculiar suppletion in the manga Azumanga Daioh where characters' hands turn into stumps when they become angry, lose physical control of their body, and/or are fantasizing or daydreaming. A similar "stumpification" occurs to another character's feet when she becomes overjoyed. The authors argue that these suppletions relate to a metaphor of LOSS OF HANDS IS LOSS OF CONTROL. A similar alteration is likely the most recognizable suppletion in JVL: "superdeformation" or the sudden conversion of a character to chibi style (chibi often is used to mean "short/small person/child"). Here, the graphic style of a figure is changed from its normal representation to an extremely cartoony style indicating a state of overwhelming emotion. Superdeformation can apply to whole figures or just parts of figures (usually the face). A less common suppletion is "paperification," where a figure will turn into paper and flutter away at times of embarrassment or deflating emotion.

Characters also take on animalistic traits in the service of conveying emotions. Long upper lips reminiscent of monkeys appear on particularly lustful or creepy men, pointed animalistic teeth appear in the mouths of angry people, and a catmouth shaped like a turned " 3 " appears for mischievous characters, sometimes accompanying the affixation of cat ears. A similar representation is used for poses like a begging dog (hands up, floppily hanging in front of the chest), again with affixed dog-ears to the head. These depictions create "blends" (Fauconnier and Turner, 1998, 2002) between the human characters and these different animals, such that particular aspects of those animals are invoked in the blend, but the characters do not become animals. For example, not all concepts related to dogs emerge when characters take on doglike attributes-just the associations with begging specifically.

Other common suppletions include schematic ways for drawing characters. Figures may assume a particular "shocked pose" with their hands up and arching their back away from the object causing surprise. Similarly, figures may also take the shape and representation of Edvard Munch's famous painting The Scream when they are surprised. In these cases, the entire body is replaced with this pose in order to convey the idea of shock.

Like the way that upfixes form a class of affixes, a class of suppletions seems to substitute various morphemes for the eyes of characters. These "eye-umlauts" (Cohn, 2013b) replace eyes with items like dollar signs, hearts, or stars to convey some sort of additional meaning beyond the range afforded by various types of iconic "eye schemas" that might include wide eyes, narrow eyes, etc. JVL uses many variations on eyes to convey meaning, such as eye-umlauts like hearts or swirls, as in American and European visual languages. However, other manipulations to eyes include roughly drawn circles, black dots, bubble eyes, glowing eyes (surrounded by a shaded upper head), and a replacement of eyes by the shape: $><$. Eyes can also be left empty, erased altogether, or "pop" out of the head. Similar manipulations occur to pupils, either shrinking them or omitting them. Given this wide variation, it is an open question as to whether these morphemes should be considered "eye-umlauts" that substitute for more common eye schema (like dollar signs), or whether these morphemes are merely included in the range of "normal" eye schema used in JVL.

Finally, some manipulations to bodies can tenuously be categorized as suppletions in that they "replace" body parts by enlarging them. For example, ears might grow to abnormal size to indicate a character is listening to a far off conversation, or mouths might grow larger than a whole face, often when a character is shocked. A more dramatic enlargement comes in 
the form of a "giant yelling head" where a character's head enlarges to an enormous size-bigger than an entire body-to yell at someone else when they are particularly angry (often disembodied, sometimes using a megaphone). This invokes the metaphor of ANGER IS HOT FLUID IN A PRESSURIZED CONTAINER since the anger becomes so pressurized that it expands the "container" of the head (Lakoff, 1992), a metaphor which appears in visual morphology across cultures' comics (Forceville, 2005; Shinohara and Matsunaka, 2009). These manipulations only somewhat can be considered suppletions, since they do not "replace" other morphemes so much as exaggerate or enlarge them to hyperbolic ends. Such changes may be more analogous to the ways that intonation can be used semantically, such as extending the vowel in loooooong to add further iconic length (Clark, 1996). However, as described previously, we view searching for a direct analogy between verbal and visual languages as ultimately less important than describing how such morphological change operates in the service of meaning.

\subsection{Reduplication}

Meaning can also change through the repetition of morphemes. In graphic form, this commonly occurs when body parts or whole figures are repeated to show a change in action within a single image. JVL commonly does this throughout many representations, especially drawing a character with two heads undergoing some type of rapid change in emotional state, like doing a "double take" or a character shaking their arms up and down frantically.

\subsection{Backgrounds}

Finally, manga often use patterned ways to draw backgrounds of a scene to aid in conveying the emotional states of characters (Shinohara and Matsunaka, 2009; Sakurai et al., 2011; Brenner, 2007). Like many visual morphemes, these schemas require experience with manga in order to be understood comprehensibly (Nakazawa, 2005; Kikuchi et al., 2005; Murata, 1994). These representations are not easily classified as morphemes in the sense of being a particular focused schema (such as manpu like hearts or a sweat-drop), but they are patterned linkages of form and meaning used repeatedly throughout books in particular contexts. They also do not have an analogue with morphological processes in other types of languages, being a facet of the visual aspects of scene representation.

There are several consistent backgrounds related to emotion. The most simple are when the background is entirely black, when the entire representation (including the characters) are shaded in grey, or when just the primary character is shaded grey, often with background omitted to be purely white. Other representations appear to extend morphemes that also might apply to character's bodies, such as hearts in a background or vertical lines depicting gloom. Schematic backgrounds also might depict fuzzy circles, sparkles, or wavy lines.

Some backgrounds involve aspects of nature or weather, such as flower petals to represent joy or love, and fire, lightning, or fog to represent anger (Shinohara and Matsunaka, 2009). These representations do not show the iconic depictions of nature or weather: There are not actually flowers in the scene, and there may not be an actual storm. Rather, these backgrounds invoke a metaphorical schema of EMOTION IS A METEOROLOGICAL FORCE THAT SURROUNDS A PERSON (Shinohara and Matsunaka, 2009), thereby connecting the emotional force associated with natural acts to the emotions of the characters. A "storm" is thus interpreted as suggesting "stormy" or "turbulent" emotions.

\section{Dialect differences in JVL morphology}

Having outlined the visual morphology across JVL generally, we next sought evidence that usage of these schemas differs across genres. Such an analysis requires a corpus analysis across several books, beyond the case studies of individual comics that have characterized previous studies (e.g., Feng and O'Halloran, 2012; Forceville, 2011; Forceville et al., 2010). Ultimately we foresee creating a large corpus whereby recognition of different visual languages and dialects will emerge from the structured patterns shown in the data itself. However, in lieu of such a massive corpora for visual languages in general and JVL specifically at present, we thus drew on 10 shonen and 10 shojo manga as a representative sample in these first efforts to investigate such issues.

Genres of manga are often delineated by the demographics of a so-called intended audience, such as shonen, shojo, seinen, and josei, which are ostensibly marketed to boys, girls, young men, and women, respectively. While these genres supposedly have intended audiences, both genders independently favour shonen manga to other genres, and boys typically do not read shojo manga (Allen and Ingulsrud, 2005). In addition, shonen manga is perceived as the stereotypical genre of manga-and also the best selling-both within and outside of Japan (Allen and Ingulsrud, 2005), while shojo manga appears to have a more targeted readership. These genres also each have their own implied or expected storylines. Shonen manga usually take on more adventurous themes involving fantasy and sci-fi worlds, sports, samurai/ ninja, or other scenarios that often revolve around physical conflict, while shojo manga typically focus on the emotional 
and psychological states of their characters, often with themes of romance, daily life, and school, but also possibly adventures (Brenner, 2007; Gravett, 2004; Schodt, 1983; Drummond-Mathews, 2010; Prough, 2010).

In terms of VLT, genres of manga have been treated as having distinct "dialects" within the broader Japanese Visual Language shared by many authors of manga. These "dialects" are defined by the ways that shonen and shojo manga appear to differ across many dimensions of structure. The most noticeable differences do include their visual styles and some aspects of morphology, but also aspects of page layouts and storytelling (which we will not discuss here).

The origins of the shonen manga style are often attributed to the "God of manga" Osamu Tezuka, and he is frequently credited with starting the shojo genre as well (Schodt, 1983, 1996). While Tezuka did appear to influence subsequent shojo manga creators, the origins of this style can actually be traced to other authors from the 1950s, particularly Makoto Takahashi, along with even earlier magazine illustrators, like Jun'ichi Nakahara from the 1930s (Takahashi, 2008). These artists laid the foundation of the stereotypical shojo style of more rounded shapes and soft lines, along with using many different "oversized" eye schemas-particularly with stars/sparkles in them-and floral backgrounds to reinforce the emotional salience of a scene (Takahashi, 2008; Prough, 2010). These elements are thought to define the character of shojo manga, and contrast from the more angular lines of shonen manga, which use morphology to reinforce actionrelated plots, such as motion lines and zoom lines (Brenner, 2007; Schodt, 1983; Takahashi, 2008). The stylistic differences unique to shojo manga in contrast to the culturally dominant shonen manga have been described as a "visual idiom. . . [used] to quietly exclude those who did not understand [girls' concerns]" (Takahashi, 2008, 129). In other words, the patterns in shojo manga attempted to differentiate one social group from another (here by gender), just like most linguistic dialects.

Before proceeding, a few caveats are in order. First, we acknowledge that genres-and their authors and readers-do not have strictly defined borders. Authors of shonen manga may also create shojo manga (as in the case of Osamu Tezuka), or may create works that tread the line between genres. It may be the case that, for these authors, their idiolects may remain steady regardless of the genre (as opposed to authors "code-switching" between dialects given their audience). Second, there are most certainly authors of manga whose drawing systems fall outside of the broader system of the Japanese Visual Language. Again, the notion of JVL-and its dialects-is an "average" of the patterns across the minds of those who use it. Some authors of manga may not use it (note again: JVL $\neq$ manga). Thus, in describing a potential "Shonen JVL" we are less attempting to delineate the boundaries of shonen or shojo manga-i.e., the actual demographics of a publishing category-but rather to describe the system that appears to be used in works that are bound by that common label. This endeavour should not be mistaken for a deterministic attempt at claiming universality across all creators and readers of manga, but rather an exploration of the systematic features found across a graphic system. Again, this is directly analogous to descriptions of linguistic systems: No description of spoken Japanese or English (or their dialects) fully encapsulates the range of the unique patterns found in the idiolects of every speaker, but rather attempts to describe the systematic patterns averaged across a population, including their patterned diversity (i.e., in "dialects").

Given all this, we used our list of 73 visual morphemes to conduct a preliminary investigation of the patterns that appear in shonen and shojo manga. We predicted that, because JVL as a visual language extends across dialects, similar visual morphology should appear in both shonen and shojo manga. However, we hypothesized that certain morphemes may be used in differing proportions between genres, and perhaps some morphemes would be unique to one genre or another. These results would reflect the differences between sub-dialects in ways that are analogous to variations in vocabulary used between varieties of linguistic types in verbal and signed languages, and would support the idea that shojo manga developed genre-specific conventions to suit its readership in ways that differed from shonen manga.

\subsection{Methods}

\subsubsection{Materials}

Our corpus consisted of 10 shonen and 10 shojo manga ranging in dates from 1997 to 2012 . These manga comprised the top 10 most popular manga series for each respective genre for May 2014 according to the website www. mangareader.net. A full list of works analyzed appears in the Appendix 2: Works Analyzed.

\subsubsection{Data analysis}

We coded for 73 individual schemas related to the representation of emotion throughout our sample manga, as described previously. Each panel was coded in our materials as either containing or not containing schemas. If a panel used schemas, each individual schema was recorded. Shonen manga averaged 52 pages per book, with around 293 schema per book. Shojo manga averaged 43 pages per book, with an average of 208 schema per book. Overall, we coded for 2711 schema across 5007 panels, distributed over 975 pages. Our analysis generated means based on the number of particular morphemes out of all morphemes of a given book. Means for each morpheme across books were then compared between genres using Independent Samples t-tests. 


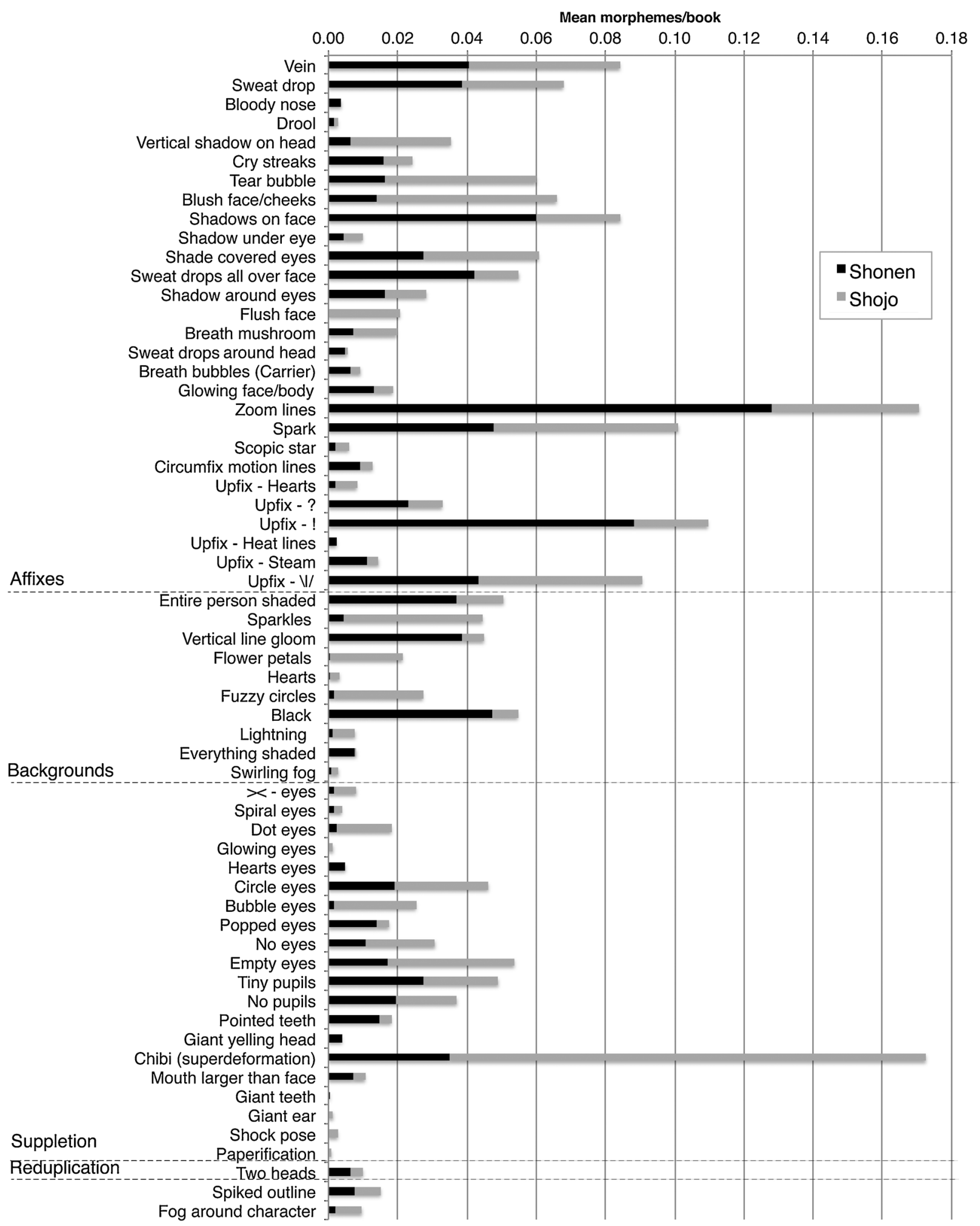

Fig. 3. Proportions of visual morphemes used in 10 shonen and 10 shojo manga. 
Table 1

Mean number of morphemes found to be significantly different in their proportion across 10 shonen and 10 shojo manga. Morphemes that were not significantly different are excluded. Grey highlight indicates which genre had higher means.

\begin{tabular}{lllll}
\hline Morphology & Morph type & Shonen (mean) & Shojo (mean) \\
\hline Bloody nose & Affix & 0.004 & 0.000 & $-1.88^{\wedge}$ \\
Shadows on face & Affix & 0.060 & 0.024 & $-1.73^{\wedge}$ \\
Sweat drops all over face & Affix & 0.042 & 0.013 & $-2.94^{*}$ \\
Zoom lines & Affix & 0.128 & 0.042 & $-2.62^{*}$ \\
Upfix -? & Affix & 0.023 & 0.010 & $-1.93^{\wedge}$ \\
Upfix-Steam & Affix & 0.011 & 0.003 & $-1.76^{\wedge}$ \\
Pointed teeth & Suppletion & 0.015 & 0.003 & $-1.95^{\wedge}$ \\
Giant yelling head & Suppletion(?) & 0.004 & 0.000 & $-2.76^{*}$ \\
Entire person shaded & Background & 0.037 & 0.014 & $-2.06^{\wedge}$ \\
Vertical line gloom & Background & 0.039 & 0.006 & $-2.93^{*}$ \\
Black background & Background & 0.047 & 0.007 & $-2.67^{*}$ \\
Everything shaded & Background & 0.008 & 0.001 & $-2.05^{\wedge}$ \\
Blush face/cheeks & Affix & 0.014 & 0.052 & $1.95^{\wedge}$ \\
Flush face & Affix & 0.000 & 0.016 \\
Dot eyes & Eye-Umlaut & 0.002 & 0.138 \\
Chibi & Suppletion & 0.035 & 0.040 \\
Sparkles & Background & 0.004 & 0.021 \\
Flower petals & Background & 0.001 & 0.026 \\
Fuzzy circles & Background & 0.002 & 0.007 \\
Lightning & Background & 0.001 & $2.84^{\wedge}$ \\
\hline
\end{tabular}

$\mathrm{df}=18$.

${ }^{\star} p<.05$.

${ }^{\wedge} p<.1$.

\subsection{Results}

Across all books, $63 \%$ of all panels contained visual morphemes (3191 of 5007 panels). Within these panels, we found 2711 schemas-a rate of 1.49 schemas per panel. These rates of morphemes per panel (when present) remained roughly the same across both shonen $(M=1.48, \mathrm{SD}=.19)$ and shojo manga $(M=1.46, \mathrm{SD}=.19)$.

Out of the 73 total schemas that we identified as appearing in JVL more broadly, 61 appeared in the books in our sample, and 51 appeared in both shonen and shojo manga. Only 10 morphemes were constrained to a single genre. Across books, the most used morphemes were superdeformation to a chibi style, zoom lines, exclamation mark and spiked upfixes, sparks floating next to character's eyes, shadows on character's faces, veins, gigantic sweat drops, and blush on the cheeks (see Fig. 3). In some cases, the proportion of these morphemes in an individual genre motivated this prevalence (chibi, zoom lines), while others were more balanced across genres (veins, gigantic sweat drops).

In addition, significant or near significant differences appeared between the usage of 21 different morphemes between genres, which are all listed in Table 1. Morphemes not listed in Table 1 were not significantly different between genres. Shonen manga used more bloody noses, shadows on the face, zoom lines, question mark and steam upfixes, pointed teeth, and giant yelling heads. In backgrounds, shonen manga also had more shading of the entire person, shading of everything, use of a black background, or use of vertical lines to depict gloom. In contrast, shojo manga used more blush on the face/cheeks, flush face (where blush extends across an absent nose), dots for eyes, and superdeformation to a chibi style. Shojo manga backgrounds also used more sparkles, flower petals, fuzzy circles, and lightning.

\section{Discussion}

This study sought a preliminary investigation of the proportions of various "visual morphemes" in the Japanese Visual Language found in the two genres of shonen (boys') and shojo (girls') manga. We found common properties shared across these genres, while still maintaining distinct patterns unique to each sample. We interpret these results as suggesting that each of these genres represents a different "dialect" of the broader abstract Japanese Visual Language shared across these systems.

First, very few visual morphemes were distinctly used by only a single genre. In our sample, only shojo manga used flush face, a shock pose, a giant ear, glowing eyes, and paperification. Meanwhile only shonen manga used a giant yelling 
head, bloody noses for lust, giant teeth, and heat line upfixes. These findings do not imply that these visual morphemes are restricted to a single genre. Rather, because less than one-sixth of all morphemes investigated appeared in only a single genre, it implies that these books share most of the same visual vocabulary.

Nevertheless, some visual morphemes were used distinctly more within each genre, and these representations appeared to be characteristic of the stereotypes of that genre. For example, the backgrounds in shojo manga using flowers, sparkles, or fuzzy circles are highly associated with the shojo manga aesthetic (Takahashi, 2008; Prough, 2010). This is also true of the super-deformed chibi style and blush/flush on characters' cheeks. These morphemes are highly characteristic of the "style" associated with shojo manga. This prevalence reinforces that these particular morphemes help define their stereotypes. In particular, it is noteworthy that shonen manga were characterized by morphemes related to lust (bloody nose), moodiness (gloom lines, shadow on face, black backgrounds), anger (pointed teeth, giant yelling head), and action (zoom lines). In contrast, shojo manga were characterized by embarrassment (flush, blush), overwhelming emotion (chibi), and joy (sparkles, flower petals). Thus, the emotional content of the morphemes used by particular genres also reflect the expected emotional themes of those works.

Yet, it is important to note that, despite being found far more prevalently in these books, few morphemes were absent from both samples. Rather, they were just proportionally lower, suggesting that the differences in JVL dialects are less about types of morphemes and more about proportion of morphemes.

The overall similarities between morphemes in shonen and shojo manga support the idea of a broader Japanese Visual Language that permeates across genres, a "Standard JVL" abstracted across the patterns in individual systems. However, the difference in proportion between visual morphemes in these genres also implies that different "dialects" do exist. These differences appear as proportional variation in the use of visual morphemes. Of course, though this study has extended beyond the scope of any prior work in this regard, the morphemes studied are only a selection by which we could characterize initial characteristics of this system. In addition, morphology may simply be one dimension by which these dialects differ. As has been proposed in broad descriptions of shonen and shojo manga (i.e., not empirical corpus analyses), we may also find variation in aspects of page layout, graphic structure (i.e., "visual style"), narrative structure, and others. How we might characterize the full differences between dialects of visual languages would incorporate the sum total of variation between systems across these structures, and future empirical research can target these different domains.

As the first systematic study of visual morphemes between genres of a visual language, this project opens up several additional questions for future research. While greatly expanding the recorded properties of JVL's lexicon, we here focused primarily on aspects of morphology related solely to emotional salience. A "complete" recording of the vocabulary of JVL would need to consist of schematic patterns for both open- and closed-class morphology, and would need to go beyond morphemes conveying emotion alone (such as the various types of motion lines, carriers, and others). Research detailing the various lexicons of visual languages of the world, and how they overlap, would thus be an important facet of cross-cultural research extending the scope of this overall endeavour.

In addition, shonen and shojo are not the only genres of manga in Japan. Will genres aimed more at adults show similar trends as books aimed at younger individuals, or will the intended age of the audience change these trends? Will these morphemes differ further in more "artistic" and "independent" works of manga that may not share the graphic structure ("style") stereotypical of JVL? Has the usage of morphemes within and across dialects changed over time? Similar questions arise with regard to manga created by individuals living outside of Japan. These works ostensibly are created using JVL and thus by association are called "manga" (Brienza, 2015). Will these works also use the same proportion of visual morphology as "native speakers" of JVL from Japan? Will they appear more like a "creole" resulting from the hybrid of JVL and American or European visual languages?

These types of questions can only be answered by dedicated corpus research, and full characterization of such systems would require a larger corpus than the sample selection used here. While we consider that our results can add substantially to the understanding of these systems, we acknowledge that for the ultimate scope of our intentions, our analysis is limited in that we only analyzed 10 books from two separate genres. Nevertheless, this sample is substantially larger than any used in previous corpus studies of comics and/or manga which primarily focus on case studies of single authors (e.g., Feng and O'Halloran, 2012; Forceville, 2011; Forceville et al., 2010). We therefore hope that this initial expanded corpus analysis can sponsor additional, larger-scale analyses across various systems in the world. Extensive corpus studies like these provide the only way to truly capture a better understanding of the patterns-and the diversitywithin and between visual languages.

\section{Appendix 1. Manga morphology}

Due to low resolution of sample images, a complete listing of JVL morphology is provided online at: http://www. visuallanguagelab.com/A/jvlmorphology.html. 


\section{Appendix 2. Works analyzed}

We list our works analyzed below. We used works both translated into English and in their original Japanese. Translations are notated with an asterisk $\left(^{*}\right)$..

\section{Shonen manga}

*Hoshino, Katsura. (2004). D. Grey-Man. Viz. Media. Chapter 1, pp. 1-54.

*Ikeda, Akihisa. (2008). Rosario + Vampire II. Viz. Media. Chapter 1, pp. 1-47.

Isayama, Hajime. (2009). Shingeki no Kyojin [Attack on Titan]. Kodansha Comics. Chapter 1, pp. 1-49.

Kato, Kazue. (2009). Ao no Exorcist. Shueisha. Chapter 1, pp. 1-65.

Kishimoto, Masashi. (1999). Naruto. Shueisha. Chapter 1, pp. 1-50.

*Kubo, Tite. (2001). Bleach. Viz. Media. Chapter 1, pp. 1-53.

Mashima, Hiro. (2006). Fairy Tail. Kodansha Comics. Chapter 1, pp. 1-72.

*Oda, Eiichiro. (1997). One Piece. Viz. Media. Chapter 1, pp. 1-50.

*Okubo, Atsushi. (2004). Soul Eater. Yen Press. Chapter 1, pp. 1-59.

Tamura, Ryuhei. (2008). Beelzebub. Shueisha. Chapter 1, pp. 1-56.

\section{Shojo manga}

*Fujiwara, Hiro. (2005). Kaicho wa Maid-sama! Tokyopop. Chapter 1, pp. 1-31.

Hazuki, Kanae. (2012). Say "I love you". Kodansha Comics. Chapter 1, pp. 1-43.

*Hino, Matsuri. (2004). Vampire Knight. Viz. Media. Chapter 1, pp. 1-48.

Koichi, Kaede. (2008). Love so Life. Hakusensha. Chapter 1, pp. 1-43.

Minami, Kanan. (2007). Kyo, Koi o Hajimemasu. Shogakukan. Chapter 1, pp. 1-39.

*Motomi, Kyosuke. (2007). Dengeki Daisy. Viz. Media. Chapter 1, pp. 1-47.

*Nakamura, Yoshiki. (2002) Skip Beat. Viz. Media. Chapter 1, pp. 1-48.

Sakurakoji, Kanoko. (2007). Black Bird. Shogakukan. Chapter 1, pp. 1-38.

Tanaka, Meca. (2007). Faster than a Kiss. Hakusensha. Chapter 1, pp. 1-42.

Toyama, Ema. (2009). Watashi ni XX Shinasai! Kodansha Comics. Chapter 1, pp. 1-41.

\section{References}

Abbott, Michael, Forceville, Charles, 2011. Visual representation of emotion in manga: loss of control is loss of hands in Azumanga Daioh Volume 4. Lang. Lit. 20 (2), 91-112. http://dx.doi.org/10.1177/0963947011402182

Abel, Jessica, Madden, Matt, 2008. Drawing Words and Writing Pictures. First Second Books, New York, NY.

Allen, Kate, Ingulsrud, John E., 2005. Reading manga: patterns of personal literacies among adolescents. Lang. Educ. 19 (4), 265-280. http://dx. doi.org/10.1080/09500780508668681

Booij, Geert, 2010. Construction Morphology. Oxford University Press, Oxford.

Brenner, R.E., 2007. Understanding Manga and Anime. Libraries Unlimited, Westport, CT.

Brienza, Casey, 2015. Global Manga: "Japanese" Comics without Japan?Ashgate Publishing, Ltd., Surrey, UK.

Clark, Herbert H., 1996. Using Language. Cambridge University Press, Cambridge, UK.

Cohn, Neil, 2010. Japanese Visual Language: the structure of manga. In: Johnson-Woods, Toni (Ed.), Manga: An Anthology of Global and Cultural Perspectives. Continuum Books, New York, pp. 187-203.

Cohn, Neil, 2013a. Beyond speech balloons and thought bubbles: the integration of text and image. Semiotica 2013 (197), 35-63. http://dx.doi. org/10.1515/sem-2013-0079

Cohn, Neil, 2013b. The Visual Language of Comics: Introduction to the Structure and Cognition of Sequential Images. Bloomsbury, London, UK.

Cohn, Neil, Maher, Stephen, 2015. The notion of the motion: the neurocognition of motion lines in visual narratives. Brain Res. 1601, 73-84. http:// dx.doi.org/10.1016/j.brainres.2015.01.018

Cohn, Neil, Paczynski, Martin, Jackendoff, Ray, Holcomb, Phillip J., Kuperberg, Gina R., 2012. (Pea)nuts and bolts of visual narrative: structure and meaning in sequential image comprehension. Cognit. Psychol. 65 (1), 1-38. http://dx.doi.org/10.1016/j.cogpsych.2012.01.003

Cohn, Neil, Jackendoff, Ray, Holcomb, Phillip J., Kuperberg, Gina R., 2014. The grammar of visual narrative: neural evidence for constituent structure in sequential image comprehension. Neuropsychologia 64, 63-70. http://dx.doi.org/10.1016/j.neuropsychologia.2014.09.018

Drummond-Mathews, Angela, 2010. What boys will be: a study of Shōnen Manga. In: Johnson-Woods, Toni (Ed.), Manga: An Anthology of Global and Cultural Perspectives. Continuum Books, New York, pp. 62-76.

Engelhardt, Yuri, 2002. The Language of Graphics (Doctoral Dissertation). FGw/FNWI: Institute for Logic, Language and Computation, University of Amsterdam.

Fauconnier, Gilles, Turner, Mark, 1998. Conceptual integration networks. Cognit. Sci. 22 (2), 133-187.

Fauconnier, Gilles, Turner, Mark, 2002. The Way We Think: Conceptual Blending and the Mind's Hidden Complexities. Basic Books, New York.

Feng, Dezheng, O'Halloran, Kay L., 2012. Representing emotive meaning in visual images: a social semiotic approach. J. Pragmat. 44 (14), 2067-2084. http://dx.doi.org/10.1016/j.pragma.2012.10.003

Forceville, Charles, 2005. Visual representations of the idealized cognitive model of anger in the Asterix album La Zizanie. J. Pragmat. 37 (1), $69-$ 88. 
Forceville, Charles, 2011. Pictorial runes in Tintin and the Picaros. J. Pragmat. 43, 875-890.

Forceville, Charles, Veale, Tony, Feyaerts, Kurt, 2010. Balloonics: the visuals of balloons in comics. In: Goggin, Joyce, Hassler-Forest, Dan (Eds.), The Rise and Reason of Comics and Graphic Literature: Critical Essays on the Form. McFarland \& Company, Inc., Jefferson.

Forceville, Charles, El Refaie, Elizabeth, Meesters, Gert, 2014. Stylistics in comics. In: Burke, Michael (Ed.), The Routledge Handbook of Stylistics. Routledge, London, pp. 485-499.

Gravett, Paul, 2004. Manga: Sixty Years of Japanese Comics. HarperCollins, New York, NY.

Gubern, Román, 1972. El lenguaje de los Comics. Peninsula, Barcelona.

Hünig, Wolfgang K., 1974. Strukturen des Comic Strip. Olms, Hildensheim.

Jackendoff, Ray, 2002. Foundations of Language: Brain, Meaning, Grammar, Evolution. Oxford University Press, Oxford.

Kennedy, John M., 1982. Metaphor in pictures. Perception 11 (5), 589-605.

Kikuchi, T., Yoshida, F., Yagi, Y., 2005. Effects of pattern and color of back lines on mood expression in Manga. In: Proceedings of Annual Conference of 69th Japanese Psychological Association, p. 607.

Koch, Walter A., 1971. Varia Semiotica. Olms, Hildensheim.

Lakoff, George, 1992. The contemporary theory of metaphor. In: Ortony, Andrew (Ed.), Metaphor and Thought. Cambridge University Press, Cambridge, UK.

McCloud, Scott, 1993. Understanding Comics: The Invisible Art. Harper Collins, New York, NY.

McCloud, Scott, 1996. Understanding manga. Wizard Mag. 56, 44-48.

Murata, N., 1994. The effects of expertization reading manga notations. Sci. Read. 38, 48-57.

Nakazawa, Jun, 1998. Development of manga notation understanding. In: Proceedings of the 9th Annual Conference of Japan Society of Developmental Psychology, p. 182.

Nakazawa, Jun, 2005. The development of manga panel reading literacy. Manga Stud. 7, 6-21.

Natsume, Fusanosuke, 1997. Manga wa naze omoshiroi no ka: sono hyogen to bunpo (Why are Manga Fascinating?: Their Expressions and Grammar). NHK Library, Tokyo, Japan.

Nöth, Winfried, 1990. Comics. In: Handbook of Semiotics. University of Indiana Press, Indianapolis, IN, pp. 472-475.

Peirce, Charles Sanders, 1931. Division of signs. In: Hartshorne, Charles, Weiss, Paul (Eds.), Collected Papers of Charles Sanders Peirce: vol. 2: Elements of Logic. Harvard University Press, Cambridge, MA, pp. 134-173.

Prough, Jennifer, 2010. Shojo Manga in Japan and Abroad. In: Johnson-Woods, Toni (Ed.), Manga: An Anthology of Global and Cultural Perspectives. Continuum, New York, pp. 93-106.

Sakurai, Sho, Narumi, Takuji, Tanikawa, Tomohiro, Hirose, Michitaka, 2011. Augmented emotion by superimposing depiction in comics. In: Proceedings of the 8th International Conference on Advances in Computer Entertainment Technology,

Schodt, Frederik L., 1983. Manga! Manga! The World of Japanese Comics Kodansha America Inc., New York.

Schodt, Frederik L., 1996. Dreamland Japan: Writings on Modern Manga. Stonebridge Press, Berkeley.

Shinohara, Kazuko, Matsunaka, Yoshihiro, 2009. Pictorial metaphors of emotion in Japanese comics. In: Forceville, Charles, Urios-Aparisi, Eduardo (Eds.), Multimodal Metaphor. Mouton De Gruyter, New York, pp. 265-293.

Takahashi, Mizuki, 2008. Opening the closed world of shojo manga. In: MacWilliams, Mark W. (Ed.), Japanese Visual Culture: Explorations in the World of Manga and Anime, pp. 114-137.

Takekuma, Kentaro, 1995. "Hito me de wakaru "keiyu" zukan" (Pictorial guide of keiyu understandable at a glance). In: Manga no yomikata (How to Read Manga). Takarajimasha EX, Tokyo, pp. 78-105.

Walker, Mort, 1980. The Lexicon of Comicana. Comicana, Inc., Port Chester, NY.

Wilson, Brent, 1999. Becoming Japanese: Manga, children's drawings, and the construction of national character. Vis. Arts Res. 25 (2), $48-60$.

Wilson, Brent, 2015. What happened and what happened next: kids' visual narratives across cultures. In: Cohn, Neil (Ed.), The Visual Narrative Reader. Bloomsbury, London, pp. 185-227. 